\title{
NMR Parameters Determination through ACE Committee Machine with Genetic Implanted Fuzzy Logic and Genetic Implanted Neural Network
}

\author{
Mojtaba ASOODEH ${ }^{1}$, Parisa BAGHERIPOUR ${ }^{1}$, and Amin GHOLAMI ${ }^{2}$ \\ ${ }^{1}$ Department of Petroleum Engineering, Aligudarz Branch, Islamic Azad University, \\ Aligudarz, Iran; e-mail: asoodeh.mojtaba@gmail.com \\ ${ }^{2}$ Abadan Faculty of Petroleum Engineering, Petroleum University of Technology, \\ Abadan, Iran
}

\section{Abstract}

Free fluid porosity and rock permeability, undoubtedly the most critical parameters of hydrocarbon reservoir, could be obtained by processing of nuclear magnetic resonance (NMR) log. Despite conventional well logs (CWLs), NMR logging is very expensive and time-consuming. Therefore, idea of synthesizing NMR log from CWLs would be of a great appeal among reservoir engineers. For this purpose, three optimization strategies are followed. Firstly, artificial neural network (ANN) is optimized by virtue of hybrid genetic algorithm-pattern search (GA-PS) technique, then fuzzy logic (FL) is optimized by means of GA-PS, and eventually an alternative condition expectation (ACE) model is constructed using the concept of committee machine to combine outputs of optimized and non-optimized FL and ANN models. Results indicated that optimization of traditional ANN and FL model using GA-PS technique significantly enhances their performances. Furthermore, the ACE committee of aforementioned models produces more accurate and reliable results compared with a singular model performing alone.

Keywords: NMR, rock physics, petrophysics, integrated intelligent systems. 


\section{INTRODUCTION}

Nuclear magnetic resonance (NMR) log contains priceless information about the in situ characteristics of hydrocarbon-bearing intervals. Free fluid porosity and permeability are two invaluable parameters of reservoir formations which could be determined by interpreting transverse relaxation time data ( $T_{2}$ distribution) of NMR log. NMR logging becomes impractical in casedhole wells and where insufficient deal of money and time exists. However, invaluable information obtained from NMR log has tempted several researchers to devise quick, cheap, and practical methods of gaining NMR log parameters. Conventional well log data have been used by some researchers for synthesizing NMR log parameters through the use of intelligent systems (Mohaghegh 2000a, Malki and Baldwin 2002, Ogilvie et al. 2002, Labani et al. 2010). These methodologies enable reservoir engineers to extract free fluid porosity and permeability for both cased holes and open holes in a cheap and fast way. Since conventional well logs are cheap and available for almost all wells, many researchers are interested in extracting petrophysical, geomechanical, and geophysical properties from conventional well logs using intelligent systems (Kadkhodaie-Ilkhchi et al. 2009, Asoodeh and Bagheripour 2012a, 2013a; Asoodeh 2013, Bagheripour and Asoodeh 2013). A growing tendency among researchers has been emerged in recent years to utilized integrated approaches for enhancing accuracy of final prediction (Sharkey 1996, Chen and Lin 2006, Asoodeh et al. 2014a, b, Gholami et al. 2013, Asoodeh and Bagheripour 2013b). However, no optimization on individual intelligent systems has been done till now. In this study, artificial neural network (ANN) and fuzzy logic (FL) are optimized stochastically by means of hybrid genetic algorithm-pattern search (GA-PS) technique to estimate NMR log parameters. This strategy significantly enhances efficiencies of ANN and FL models. Owing to further increase in accuracy of final prediction, an alternative condition expectation (ACE) committee with GA-PS optimized FL and ANN models is constructed. This model is capable of satisfyingly improving accuracy of NMR $\log$ prediction using the concept of committee machine. Several papers have investigated performance of committee machine compared with individual intelligent systems performing alone (Asoodeh and Bagheripour 2013b, Afshar et al. 2014, Asoodeh and Kazemi 2013). The proposed strategy was applied in one of Iranian hydrocarbon fields with carbonate reservoir formations. A satisfying improvement was observed in accuracy of predicted NMR parameters, including free fluid porosity and permeability after three-step optimization approach.

\section{METHODS}

This study follows a three-step strategy for formulating conventional well logs to NMR log parameters. The first step is devoted to optimization of arti- 


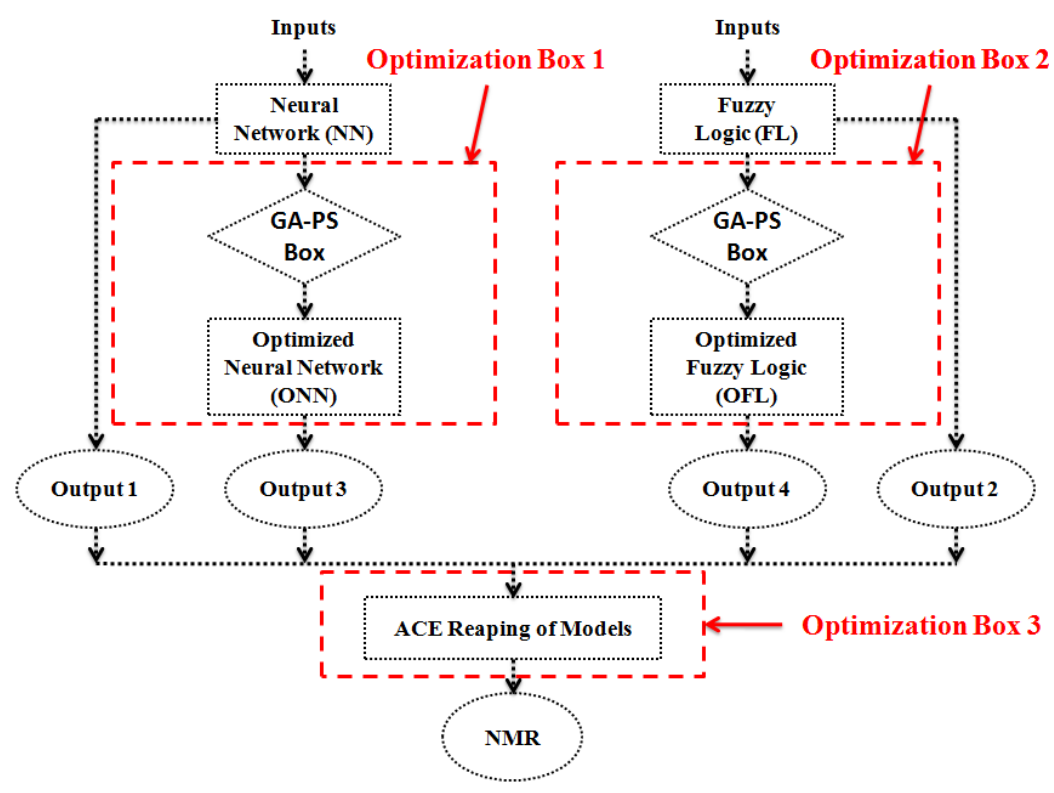

Fig. 1. General flowchart followed in this study.

ficial neural network (ANN) by means of hybrid genetic algorithm-pattern search (GA-PS) technique. Next, fuzzy logic (FL) model is optimized with GA-PS tool. Up to here, four models, including optimized FL, optimized ANN, non-optimized FL, and non-optimized ANN exist. In last stage of this study, an alternative condition expectation (ACE) committee of four models is constructed to combine their results. This step will reap benefits of all models and will enhance accuracy of final prediction. A flowchart of this study is provided in Fig. 1.

\subsection{Artificial neural network}

Artificial neural networks emulate the biological neural storage and analytical operations of brain through non-linear processing elements (neurons) connected by fixed (Lippmann 1987), variable (Barhen et al. 1989), or fuzzy (Gupta and Ding 1994) weights. Each neural network is made up of three layers (parallel arrangement of neurons): an input layer, one or more hidden layers depending on complexity of the problem, and an output layer. Backpropagation is a popular learning method for neural networks. It requires a set of input/output training data to gain knowledge from them and then applying that knowledge to new situations and problems. This method performs in a way that initial weights are randomly assigned to the neural network and its output is then calculated through random weights and biases. 
Differences between neural network's outputs and desired outputs are evaluated and the obtained error is then propagated back to the neural network to adjust connection weights and biases. This process is conducted iteratively until network's output locates in close proximity of desired output.

\subsection{Fuzzy logic}

The basic theory of fuzzy logic was proposed by Zadeh in 1965 as an extension of traditional Boolean logic (Zadeh 1965). A fuzzy set is characterized by a membership function which assigns to each object a grade of membership ranging between zero and one (Zadeh 1965). The main part of fuzzy logic is the fuzzy inference system, which formulates inputs to an output. A fuzzy inference system consists of five major steps: fuzzification of input variables, application of fuzzy operators (AND, OR, and NOT) in the rule's antecedent, implication from the antecedent to the consequent, aggregation of consequent across the rules, and defuzzification. These steps have been discussed in a number of papers (Mohaghegh 2000c, Kadkhodaie-Ilkhchi et al. 2006). In the Takagi Sugeno fuzzy inference system (the most popular type of fuzzy inference system), membership functions are defined by subtractive clustering process, which divides the input-output data space into fuzzy clusters, each representing a specific part of the system behavior. Each membership function generates a set of fuzzy "if-then" rule for formulating inputs to output. The fuzzy system makes a sum of all "then" parts and uses a defuzzification method to give the final output.

\subsection{Hybrid genetic algorithm-pattern search technique}

Genetic algorithm is a stochastic global optimization tool which emulates the biological process of natural evolution for solving problems in widespread fields. It utilizes a specific terminology for optimization purpose, i.e., the function meant to optimize is called fitness function, the probable solutions of the function are called chromosomes, involved parameters in a solution are called genes, and mathematical functions which provide a stochastic generation of new solutions are called genetic operations. Genetic algorithm starts with a population of randomly generated chromosomes and evolves toward better chromosomes by applying genetic operators. This strategy provides a stochastic search capability for genetic algorithm. Evolutionary process by use of genetic operations continues until it finds the best chromosome (or solution) in which global minimum of fitness function has been occurred. More description about genetic algorithm can be found in Mohaghegh (2000b).

In the pattern search technique, the algorithm searches a set of points, called a mesh, around the current point (the point computed at the previous 
step of the algorithm). The mesh is formed by adding the current point to a scalar multiple of a set of vectors called a pattern. If the pattern search algorithm finds a point in the mesh that improves the objective function at the current point, the new point becomes the current point at the next step of the algorithm (MATLAB user's guide; Mathworks 2011).

\subsection{Alternative condition expectation (ACE)}

Alternating conditional expectation (ACE) is a nonparametric method, which was first established by Breiman and Friedman (1985). ACE is robust data driven technique widely utilized for identifying the complicated relationship between independent variables and response variable, especially in the situation when there are no prior assumptions of functional form for expressing relation between input/output data space. This method seeks the optimal transformations of response and a set of predictor variables, thereby the maximal multiple correlation between independent variables and the response variable is achieved. The optimal transformation is determined through minimizing the error variance between the transformed dependent variable and the sum of the transformed predicted variables. The value of error variance is minimized through employing of a series of single-function minimizations. Through iterative minimization of error variance, the optimal transformation of input and output is computed. Indeed, this method involved two operations for solving regression problems including conditional expectation and iterative minimization process. This technique gained increasing attention in both science and engineering fields due to its simplicity and its successful results in uncovering the nonlinearity relationship between dependent and independent variables (Malallah et al. 2006, Xue et al. 1996, Shokir 2007). For comprehensive study of ACE formulation principles, readers are referred to original paper of Breiman and Friedman (1985). In this study ACE is employed as nonlinear combiner to computing the contribution of different predictive models in overall prediction.

\section{WHY OPTIMIZATION IS NEEDED?}

\subsection{For fuzzy logic}

Subtracting clustering is an effective approach for determining the number of rules in Takagi Sugeno fuzzy model (Al-Jarrah and Halawani 2001), which reduces computation compared with mountain clustering (Bataineh et al. 2011). However, it has the flaw of being affected by constant clustering radius. It means, by use of subtractive clustering, no modification is done over spread of Gaussian membership functions and all extracted membership functions have the same variance value. Furthermore, subtractive clustering looks for cluster centers among the data points; however, the actual cluster 
centers are not necessarily located at one of data points (Bagheripour and Asoodeh 2013). These shortcomings of subtractive clustering entail to propose an alternative for setting up fuzzy rules and clusters. Stochastic optimization capability of hybrid genetic algorithm-pattern search tool provides a powerful search engine which is capable of finding optimal values of cluster centers and spreads, regardless of the above-mentioned limitations of subtractive clustering.

\subsection{For artificial neural network}

As mentioned in Section 2.1, back-propagation algorithm is the widely-used method of determining connection weights. Performance of neural network is strongly dominated by initial weights and biases which are randomly assigned to it by back-propagation algorithm. It means, the final stage of convergence depends on where the back-propagation algorithm starts from. In other words, several runs of back-propagation algorithm with similar initial condition will converge to the same minimum. By change of the initial condition, final results of neural network might differ. Therefore, to find the optimal initial condition which leads to reaching global minimum, too many boring runs of back-propagation algorithm are needed. Nonetheless, during all these runs, neural network is highly at risk of trapping in local minima. Stochastic search capability of hybrid genetic algorithm-pattern search tool is independent of initial condition of its run. It will converge to global minimum regardless from where it starts (Conn et al. 1991). Therefore, the use of hybrid genetic algorithm-pattern search tool instead of back-propagation algorithm in the structure of neural network will improve the accuracy of modeling and eliminate the probability of sticking in local minima.

\section{INPUTS-OUTPUTS DEPENDENCY}

The first criterion in modeling by intelligent system is the quality of data. Therefore, low quality data should be removed before modeling. For this purpose, a quality control was done and bad hole intervals were removed by processing of well log data. Existence of logical relationship between inputs and outputs is another important criterion which should be kept in mind while modeling. Introducing some input parameters which have no dependency with outputs, makes the intelligent system confused and weakens the modeling. Outputs of this study are free fluid porosity and permeability. Free fluid porosity includes the pores containing fluids which are expected to flow. Permeability is defined as the capacity of a rock or sediment for fluid transmission, and is a measure of the relative ease of fluid flow under pressure gradients. Therefore, conventional well logs that have an underlying dependency with these two parameters are desired. Inputs for prediction of 
free fluid porosity and permeability are the same. Because, behavioral physics of free fluid porosity and permeability are completely intertwined. Free fluid porosity increases as permeability increases and vice versa. Available well logs for this study were including DT, RHOB, NPHI, RT, PEF, GR, and Caliper. Caliper log was firstly used to identify bad hole intervals where standoff is higher than 1.5 inches in $8.5^{\prime \prime}$ hole. Bad hole intervals were removed because they contain erroneous recordings. Photoelectric factor (PEF) is a litho-log which determines composition of reservoir rocks. NMR $\log$ is independent of lithology. Therefore, PEF was not used as input. Gamma ray (GR) is an indicator of shale content. However, in unconventional carbonate reservoirs, GR is contaminated by digenesis factors and is misleading. In this study, four conventional well logs, including sonic transit time (DT), bulk density (RHOB), neutron porosity (NPHI), and true formation resistivity (RT) were chosen as inputs. These are known appropriate in-
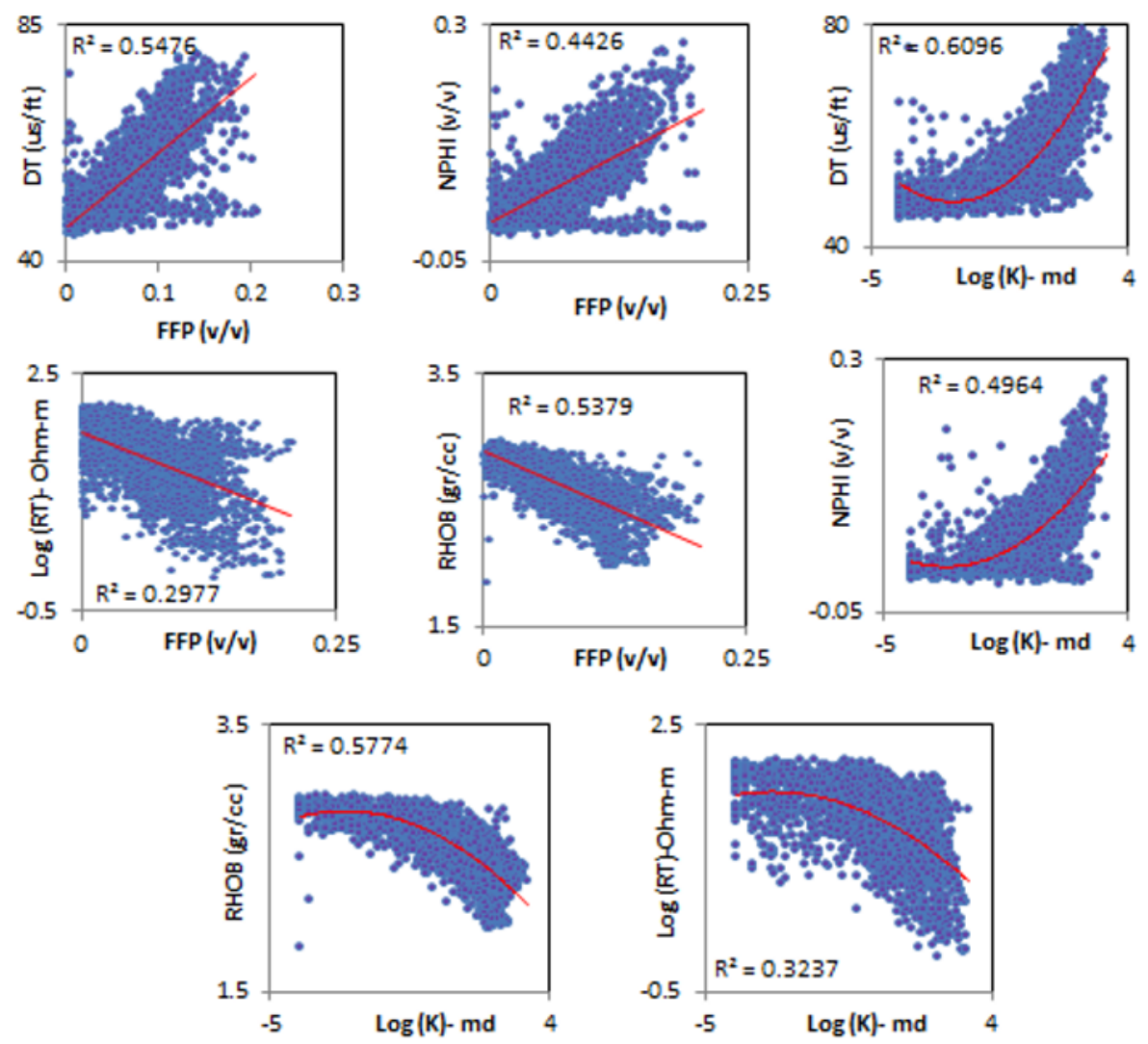

Fig. 2. Crossplots showing relationship between conventional well log data and NMR log parameters, including free fluid porosity and permeability. 
put logs having logical relationship with outputs, because the first three logs are porosity logs and the last one is attributed to fluid flow pass (tortuosity) (Asoodeh and Bagheripour 2012a). Figure 2 shows the logical dependency of chosen conventional well log data and nuclear magnetic resonance log parameters using the concept of correlation coefficient. Appendix provides conversion factors from field units to SI units for permeability and sonic transit time. Conventional well logs and NMR logs which have been used for both training and testing of models are originated from Asmari formation. Asmari formation is composed of fossilifrous limestone, dolomitic limestone, argilious limestone, sandstone, and shale and constitutes the major reservoir rock for several carbonate oil fields of Iran.

\section{MODELING, RESULTS, AND DISCUSSION}

\subsection{Optimization of artificial neural network}

At the first stage, a three-layered structure of ANN was used for estimation of free fluid porosity and permeability from conventional well logs. This structure utilizes TANSIG and PURELIN transfer functions for its hidden and output neurons, correspondingly. TANSIG is mathematically equivalent to tangent hyperbolic function and PURELIN is a simple linear function of $f(x)=x$ (Asoodeh and Bagheripour 2012b). "Trainlm" training function was used for ANN to learn from training data (for more details about Trainlm refer to Hagan and Menhaj (1994)). To achieve optimal number of hidden neurons, different ANNs with different number of hidden neurons were constructed and performance of each was evaluated using mean square error function as shown in Fig. 3. Results showed that if seven and three nodes were inserted in the hidden layer of neural network for permeability and free fluid porosity, respectively, the highest performances are achieved. The constructed model was then evaluated by means of blind testing. A set of unseen well $\log$ data was used to assess performance of constructed ANN. Results showed that the correlation coefficient between predicted values using neural networks and NMR parameters was equal to 0.84 and 0.857 for permeability and free fluid porosity, respectively. Mean square error of prediction was equal to $0.73\left[\mathrm{md}^{2}\right]$ and 0.000501 for permeability and free fluid porosity, correspondingly. In the next stage, the neural network is optimized through the search capability of hybrid genetic algorithm-pattern search tool. This procedure is discussed in the following paragraphs.

Stochastic search capability of hybrid genetic algorithm-pattern search tool enables it to escape from local minima and converge to global minimum. This feature of hybrid genetic algorithm-pattern search tool could be employed to eliminate the flaw of neural networks which use backpropagation algorithm for finding their weights. To extract the optimum val- 

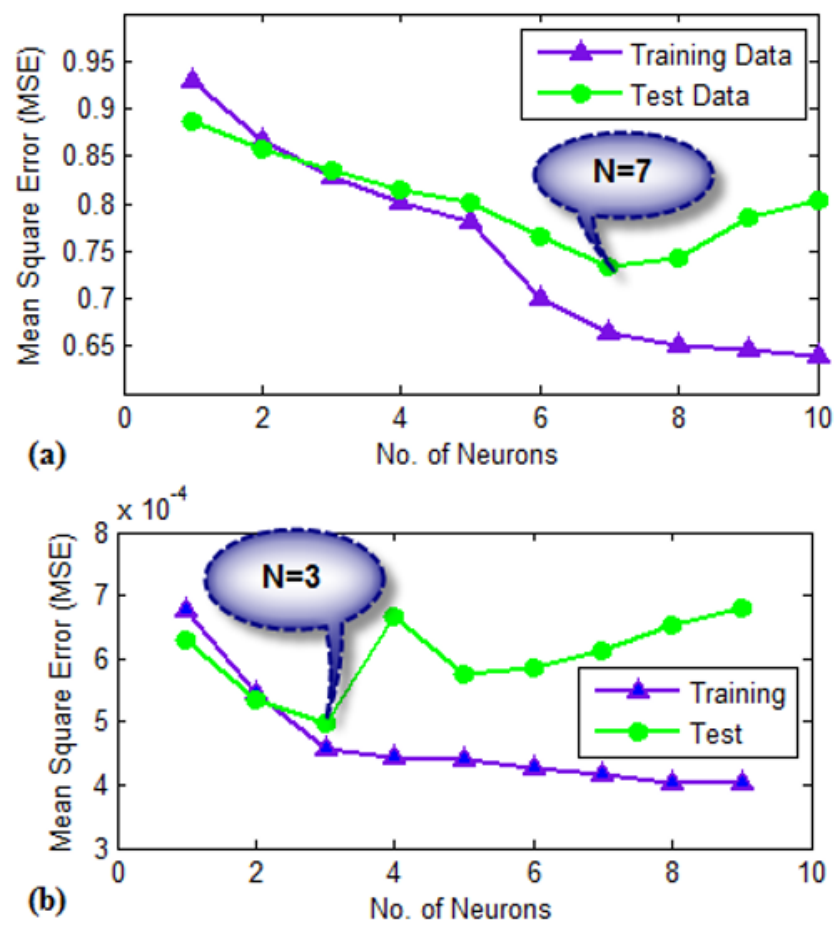

Fig. 3. Graph showing the mean square error (MSE) performance of backpropagation neural network in test data versus different number of nodes of hidden layer for: (a) permeability, and (b) free fluid porosity. Optimal models are achieved when 3 and 7 nodes are inserted in hidden layer of neural network for free fluid porosity and permeability, respectively.

ues of ANN's weights, the following mean square error function was introduced into hybrid genetic algorithm-pattern search tool:

$$
\mathrm{MSE}_{\mathrm{ONN}}=\frac{1}{K} \sum_{\ell=1}^{k}\left(\mathrm{Output}_{\mathrm{NMR} \ell}-\mathrm{Output}_{\mathrm{ONN} \ell}\right)^{2},
$$

where $\mathrm{O}_{\text {estNN }}$ is ANN output. Output ${ }_{\mathrm{ONN}}$ is estimated values of nuclear magnetic resonance (NMR) log parameters from optimized neural network, Output $_{\mathrm{NMR}}$ is the NMR log parameter, MSE $\mathrm{ONN}_{\mathrm{N}}$ is the mean square error function of optimized neural network, and $k$ is the number of training data. The process of producing an output from neural network is discussed by Asoodeh and Bagheripour (2013c). Hybrid genetic algorithm-pattern search technique looks for optimum values of connection weights of ANN such that Eq. 1 converges to its global minimum. Blue lines of Fig. 4 indicate mean value of mean square error (MSE) for a population of chromosomes (solutions) dur- 


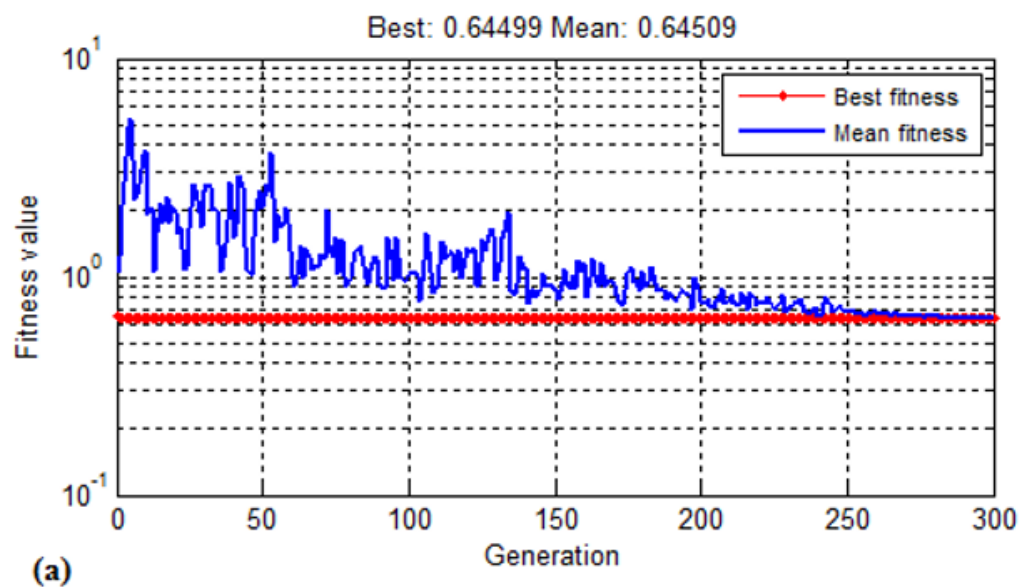

(a)

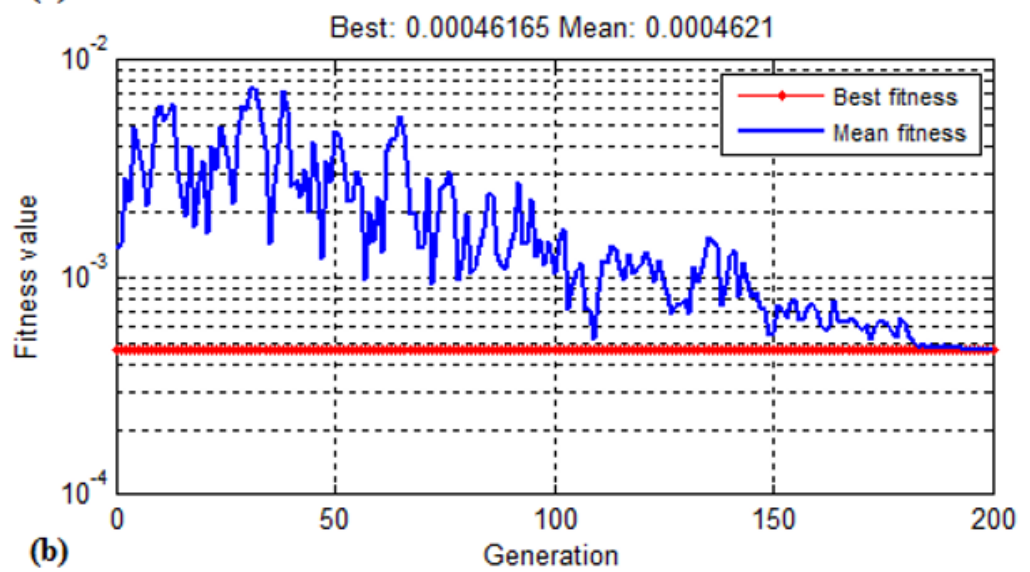

Fig. 4. Run of hybrid genetic algorithm-pattern search tool for stochastic optimization of neural network. This plot shows the best and mean fitness values of fitness functions after 300 generations for: (a) permeability and (b) free fluid porosity.

ing 300 generations for permeability model and 200 generations for free fluid porosity (FFP) models. The red lines in Fig. 4 show mean square error corresponding to best chromosomes. After stochastic optimization of neural network, performances of optimized models were evaluated by test data, i.e., test data were introduced to optimized model and quality of estimation was assessed. Figure 5 indicates error distributions for optimized and nonoptimized neural network models. It is obvious that stochastic optimization of neural networks led mean of error distributions to approach zero and standard deviation of error distributions to decrease. Furthermore, correlation coefficient of prediction has improved after stochastic optimization of neural networks. 

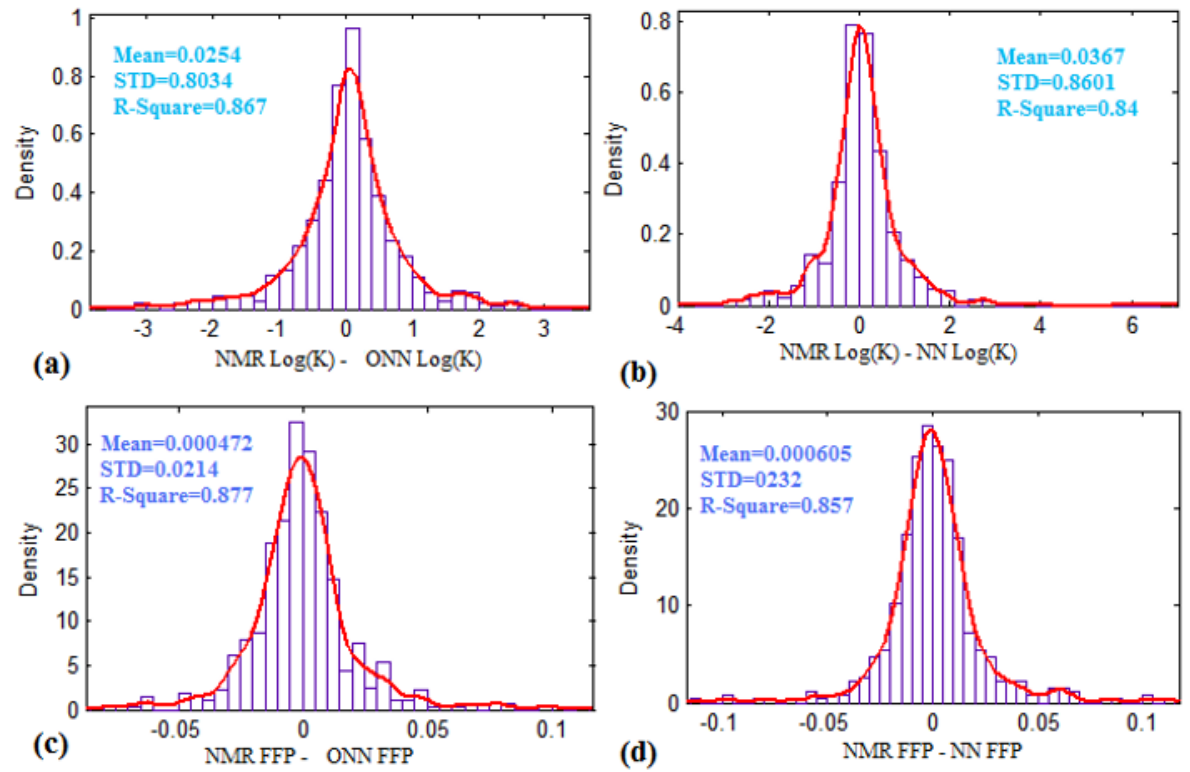

Fig. 5. Error distribution of: (a) optimized neural network for permeability, (b) nonoptimized neural network for permeability, (c) optimized neural network for free fluid porosity, and (d) non-optimized neural network for free fluid porosity. In optimized models, the mean of error distributions approach zero and their standard deviation decrease. It means, optimized models performed better than non-optimized models.

\subsection{Optimization of fuzzy logic}

The next stage of this study was initiated with construction of fuzzy model for estimation of nuclear magnetic resonance (NMR) log parameters, including free fluid porosity and permeability from conventional well log data. To find optimum fuzzy model which best describes quantitative formulation between inputs and outputs, several fuzzy inference systems with various values of clustering radius were constructed. Fuzzy inference systems which have the lowest mean square error for the test data were chosen as optimal ones. Figure 6 indicates variations of mean square error for test and training data in conjugation with variation of number of rules for different clustering radii. This figure indicates fuzzy inference systems with clustering radii of 0.6 and 0.9 for permeability and free fluid porosity, respectively, can extract the best quantitative relationship between inputs and outputs. Furthermore, this figure shows that four and two linguistic rules can in turn handle the relationship between inputs and outputs for permeability and free fluid porosity models. Figure 7 shows the extracted Gaussian membership functions 


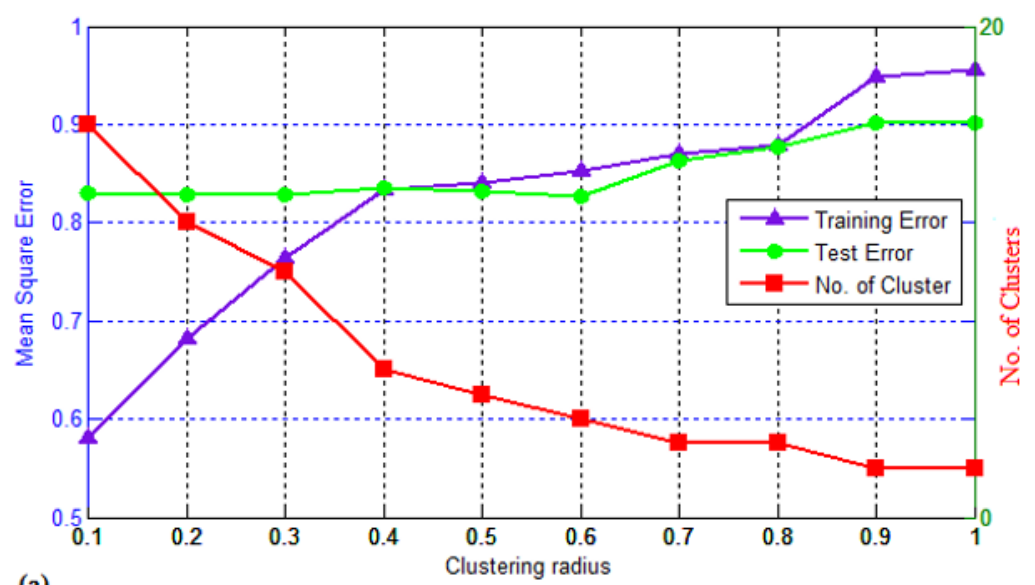

(a)

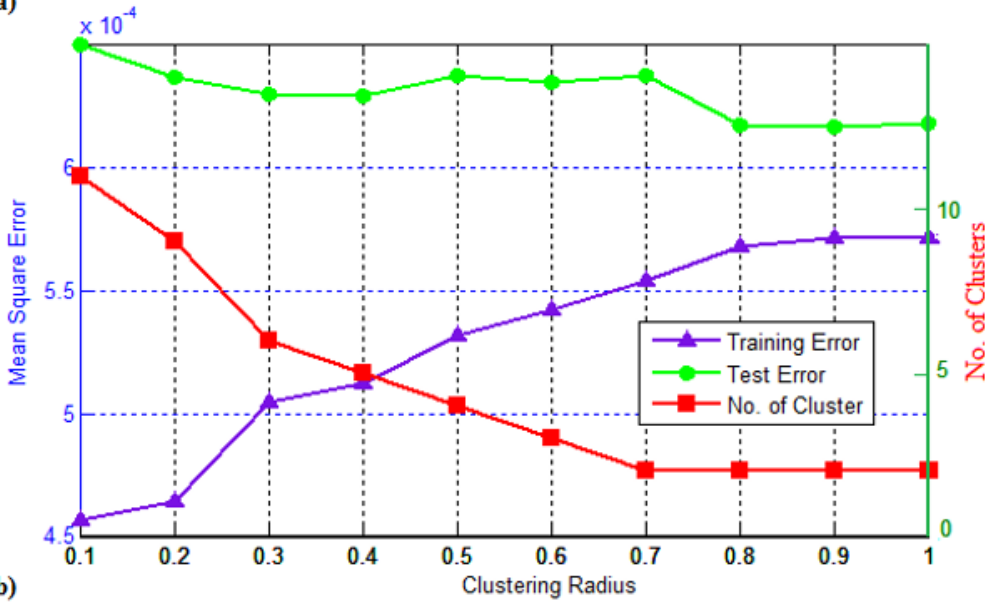

Fig. 6. Graphs showing the mean square error (MSE) performance of Takagi-Sugeno fuzzy inference system (TS-FIS) in test and training data in conjugation with number of rules versus clustering radius for: (a) permeability, and (b) free fluid porosity. Optimal models are achieved when 0.6 and 0.9 are chosen for clustering radius of permeability and free fluid porosity models, respectively.

corresponding to aforementioned linguistic rules. As this figure shows, the subtractive clustering implies no optimization over variances (spreads) of fuzzy clusters corresponding to each input. This flaw is eliminated in next step. Evaluating with test data, the results showed that correlation coefficient between fuzzy logic predicted free fluid porosity and NMR values was equal to 0.852 . This value for permeability was equal to 0.845 . Mean square errors of fuzzy logic predictions are equal to 0.000521 and $0.7201\left[\mathrm{md}^{2}\right]$ for free fluid porosity and permeability, correspondingly. 

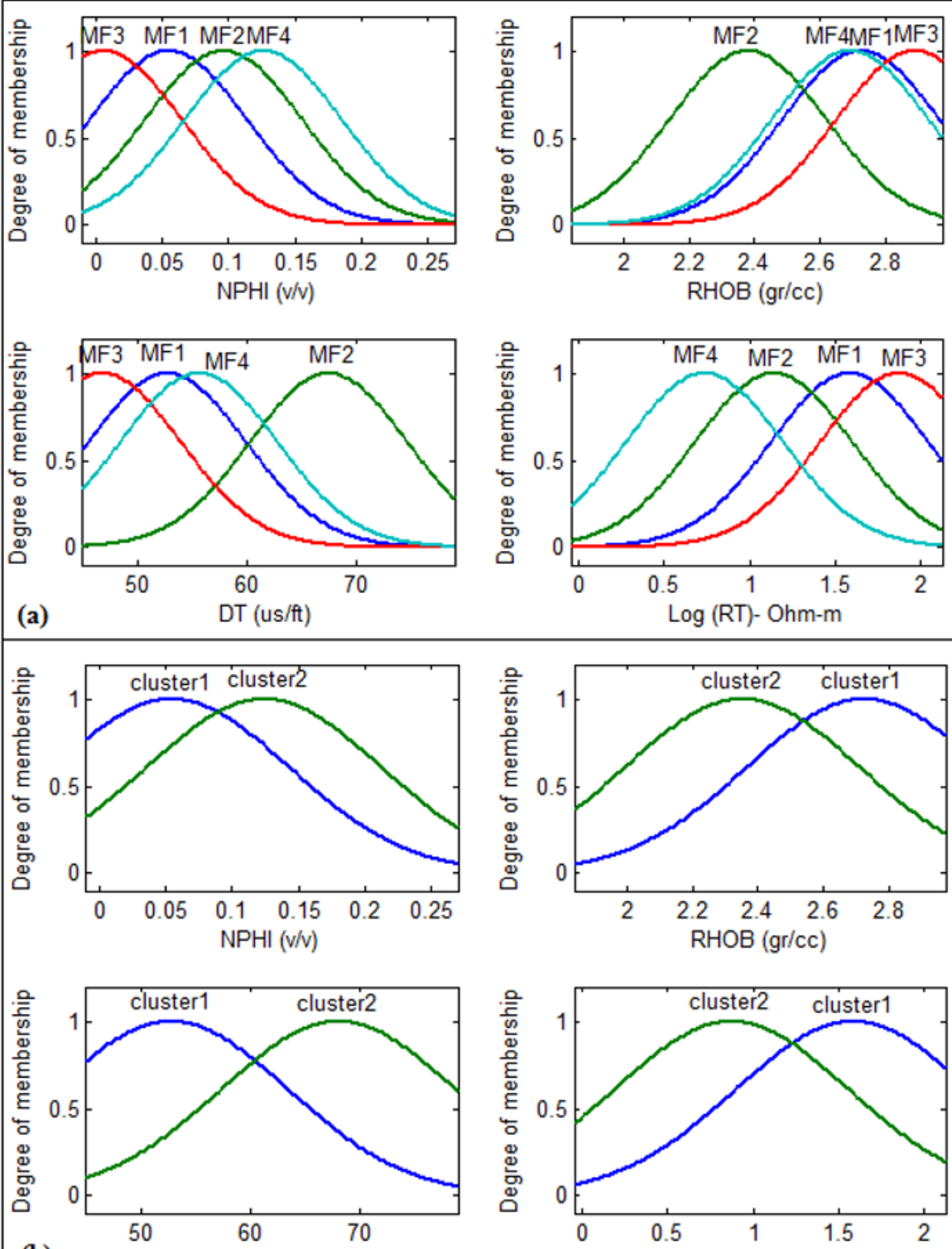

(b)

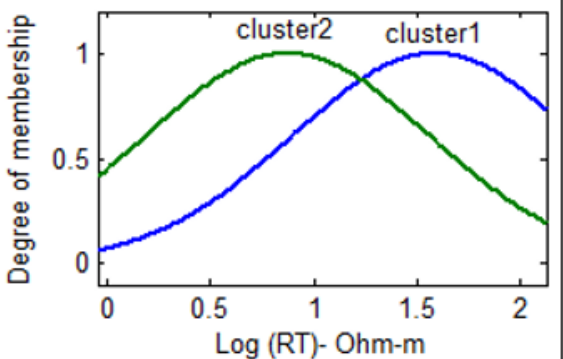

Fig. 7. TS-FIS generated Gaussian membership functions (fuzzy clusters) for the inputs of model meant to predict: (a) permeability, and (b) free fluid porosity.

Since conventional well log data, which constitute input data space, cover various ranges of data, all inputs were normalized to improve the performance of search capability of hybrid genetic algorithm-pattern search tool 
for optimizing fuzzy logic model. Since this type of normalization does not destroy the consistency of input data, shape of membership functions will just be scaled and normalization would not have any effect in the overall shape of membership functions. Similar to ANN, a mean square error (MSE) function was used as fitness function of hybrid genetic algorithm-pattern search to find optimal membership functions such that MSE function reaches its global minimum. Stochastic search capability of hybrid genetic algorithm-pattern search tool makes it possible to optimize both mean and variance (spread) of input Gaussian membership functions along with optimization of output linear membership functions. Figure 8 shows results of hybrid genetic algorithm-pattern search runs for permeability and free fluid porosity. Extracted fuzzy Gaussian membership functions are illustrated in Fig. 9. A comparison between Figs. 7 and 9 shows that the proposed
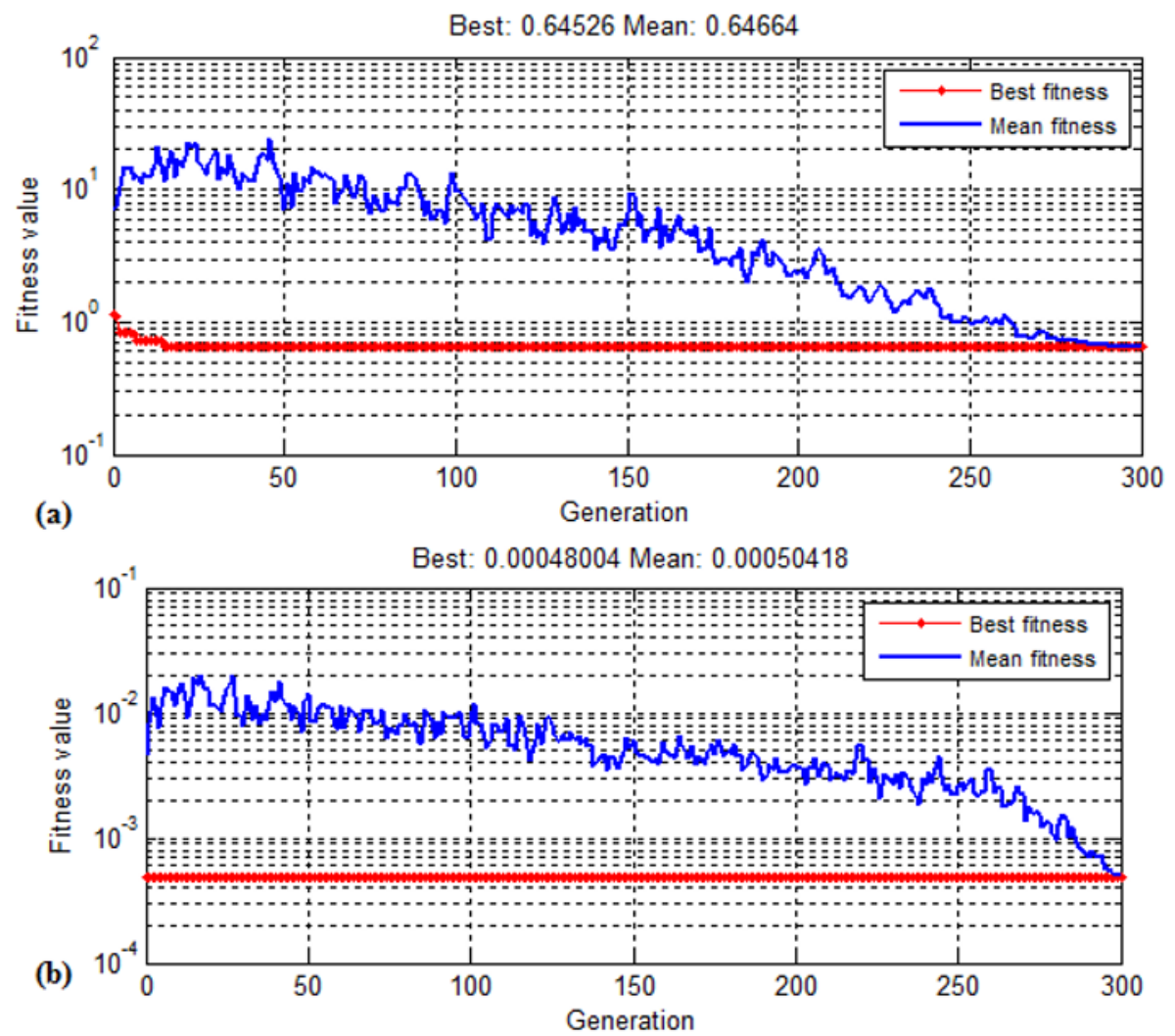

Fig. 8. Run of hybrid genetic algorithm-pattern search tool for stochastic optimization of fuzzy logic. This plot shows the best and mean fitness values of fitness functions after 300 generations for: (a) permeability, and (b) free fluid porosity. 


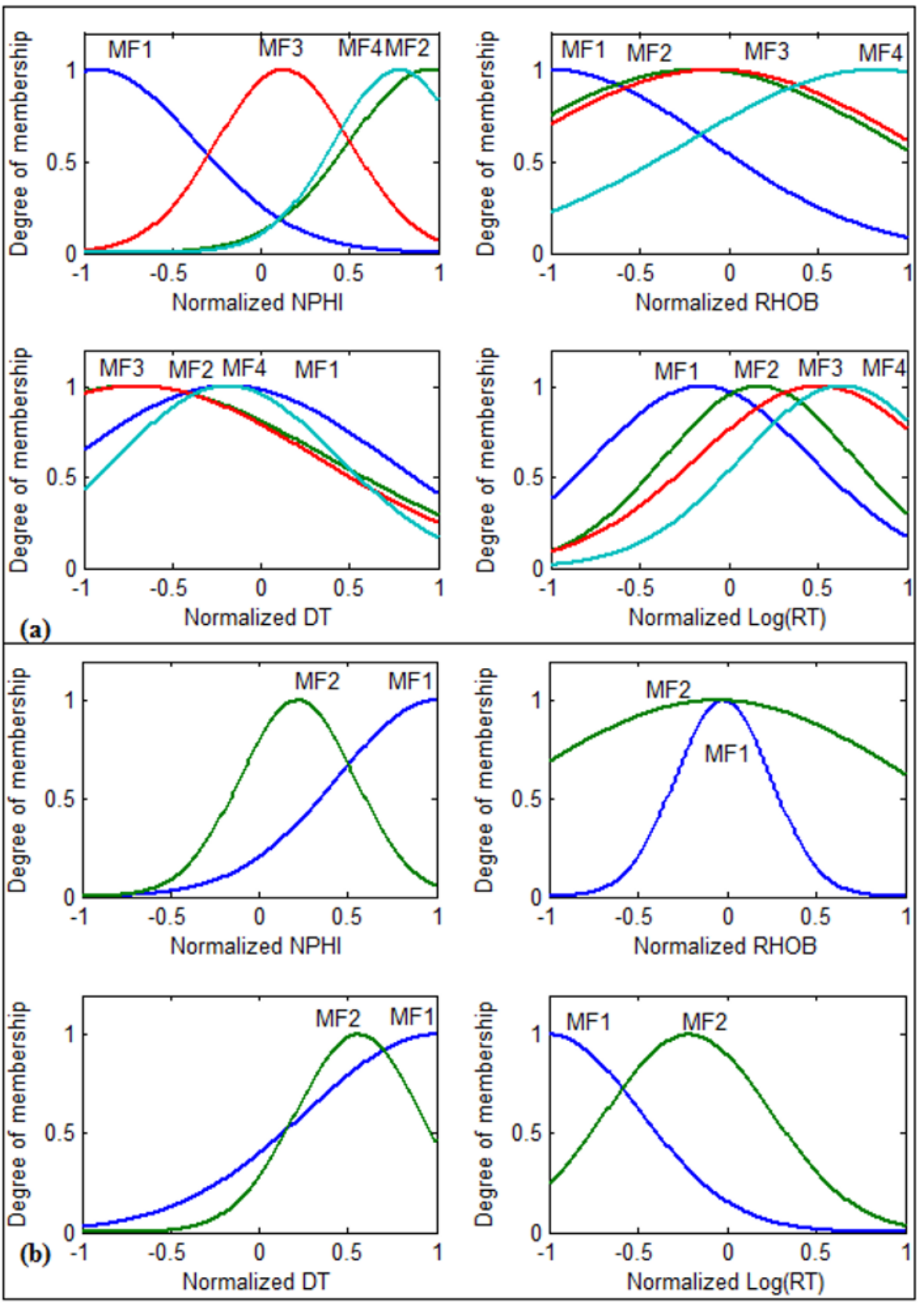

Fig. 9. Stochastically generated Gaussian membership functions (fuzzy clusters) for the inputs of model meant to predict: (a) permeability, and (b) free fluid porosity. 

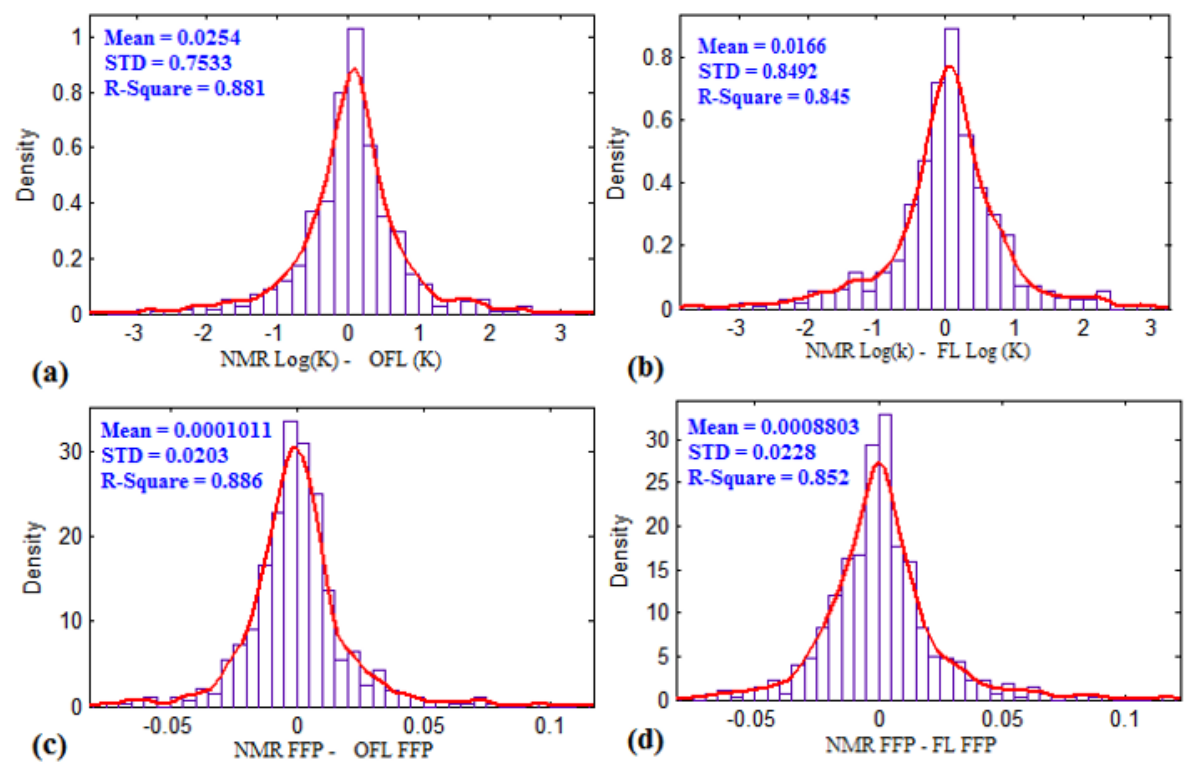

Fig. 10. Error distribution of: (a) optimized fuzzy model for permeability, (b) nonoptimized fuzzy model for permeability, (c) optimized fuzzy model for free fluid porosity, and (d) non-optimized fuzzy model for free fluid porosity. In optimized models, mean of error distributions approach to zero and their standard deviation decrease. It means, optimized models performed better than non-optimized models.

model was capable of optimizing variances (spreads) of Gaussian membership function in addition to optimizing mean of Gaussian membership functions. Figure 10 illustrates error distributions of optimized and nonoptimized fuzzy logic models. It is obvious that stochastic optimization of fuzzy logic models has reduced the absolute mean and standard deviation of error distributions. Furthermore, correlation coefficient of prediction improved after stochastic optimization of fuzzy logic models.

\subsection{ACE committee of models}

As mentioned previously, ACE is employed as a combiner to integrate outputs of different models and consequently reaping their benefits through constructing a more accurate model for estimating free fluid porosity and permeability. For this purpose, ACE transfers input parameters and target values into a desired space where maximum correlation exists between them. Thus, outputs of predictive models, including fuzzy logic, optimized fuzzy logic, neural network, and optimized neural network are employed as inputs of ACE and free fluid porosity and permeability are considered as ACE out- 


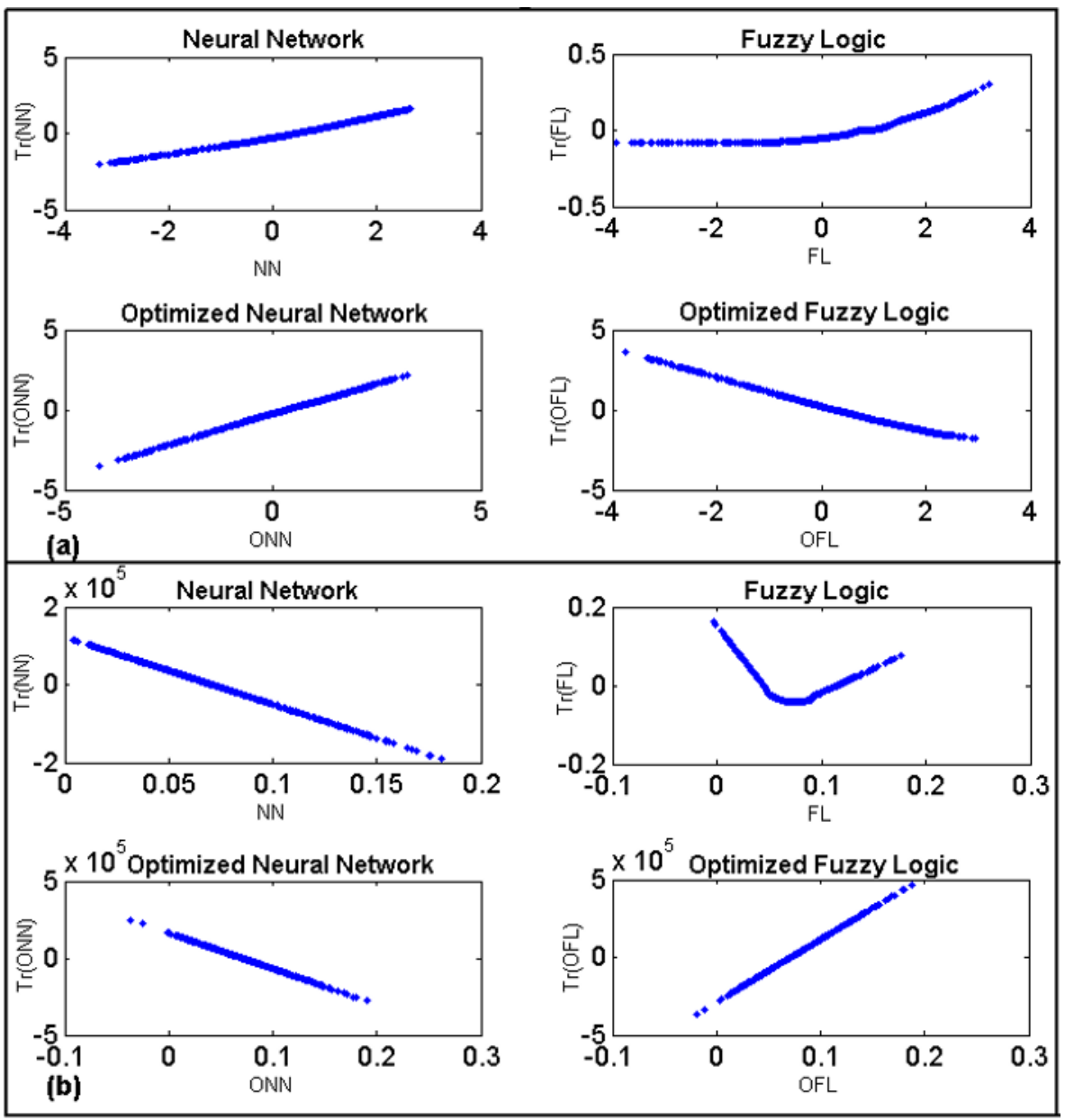

Fig. 11. Optimal transformation of input parameters which determined by ACE for: (a) permeability, and (b) free fluid porosity.

puts. Figure 11 demonstrates ACE extracted optimal transformation of input parameters for free fluid porosity and permeability, respectively. Although ACE algorithm approximates optimal transformations of input/output data space, it does not present a computational model for relating parameters and their optimal transformations. Hence, a simple polynomial function is fitted for making formulation between the parameters and their optimal transformations. The resulting polynomial functions with associated parameters for transforming neural network, optimized neural network, fuzzy logic, and optimized fuzzy logic are shown in Eqs. 2-9. 
(a) Transformations for permeability modeling

$$
\varphi^{*}(N N)=a_{2}(N N)^{2}+a_{1}(N N)+a_{0},
$$

where $\quad a_{2}=0.034882108437520, \quad a_{1}=0.0626864203614067, \quad$ and $a_{0}=-0.28077401469906$.

$$
\varphi^{*}(F L)=a_{3}(F L)^{3}+a_{2}(F L)^{2}+a_{1}(F L)+a_{0},
$$

where $\quad a_{3}=0.001772160478232$, $a_{2}=0.016384002242114$, $a_{1}=0.042023493356332$, and $a_{0}=-0.050567771173985$.

$$
\varphi^{*}(O N N)=a_{1}(O N N)+a_{0},
$$

where $a_{1}=0.756690942653011$, and $a_{0}=-0.263928319591898$.

$$
\varphi^{*}(O F L)=a_{3}(O F L)^{3}+a_{2}(O F L)^{2}+a_{1}(O F L)+a_{0},
$$

where

$$
a_{3}=0.007540599087205 \text {, }
$$

$a_{2}=0.034647276929447$, $a_{1}=-0.871553938494339$, and $a_{0}=0.211074313346156$.

\section{(b) Transformations for FFP modeling}

$$
\varphi^{*}(N N)=a_{1}(N N)+a_{0},
$$

where $a_{1}=-1735498.432421821$, and $a_{0}=122908.214346089$.

$$
\varphi^{*}(F L)=a_{4}(F L)^{4}+a_{3}(F L)^{3}+a_{2}(F L)^{2}+a_{1}(F L)+a_{0},
$$

where $\quad a_{4}=-1179.828742552175, \quad a_{3}=244.364360922208$, $a_{2}=18.984460901200$, $a_{1}=-5.166180545100$, and $a_{0}=-0.050567771173985$.

$$
\varphi^{*}(O N N)=a_{1}(O N N)+a_{0},
$$

where $a_{1}=-2324155.414675274$, and $a_{0}=163629.816053569$.

$$
\varphi^{*}(O F L)=a_{1}(O F L)+a_{0},
$$

where $a_{1}=4013501.320037709$, and $a_{0}=-286489.622011131$.

Summations of optimal transformation of input parameters are as follows:

$$
\begin{gathered}
\varphi^{*}(\log (K))=\varphi^{*}(N N)+\varphi^{*}(F L)+\varphi^{*}(O N N)+\varphi^{*}(O F L), \\
\varphi^{*}(F F P)=\varphi^{*}(N N)+\varphi^{*}(F L)+\varphi^{*}(O N N)+\varphi^{*}(O F L) .
\end{gathered}
$$



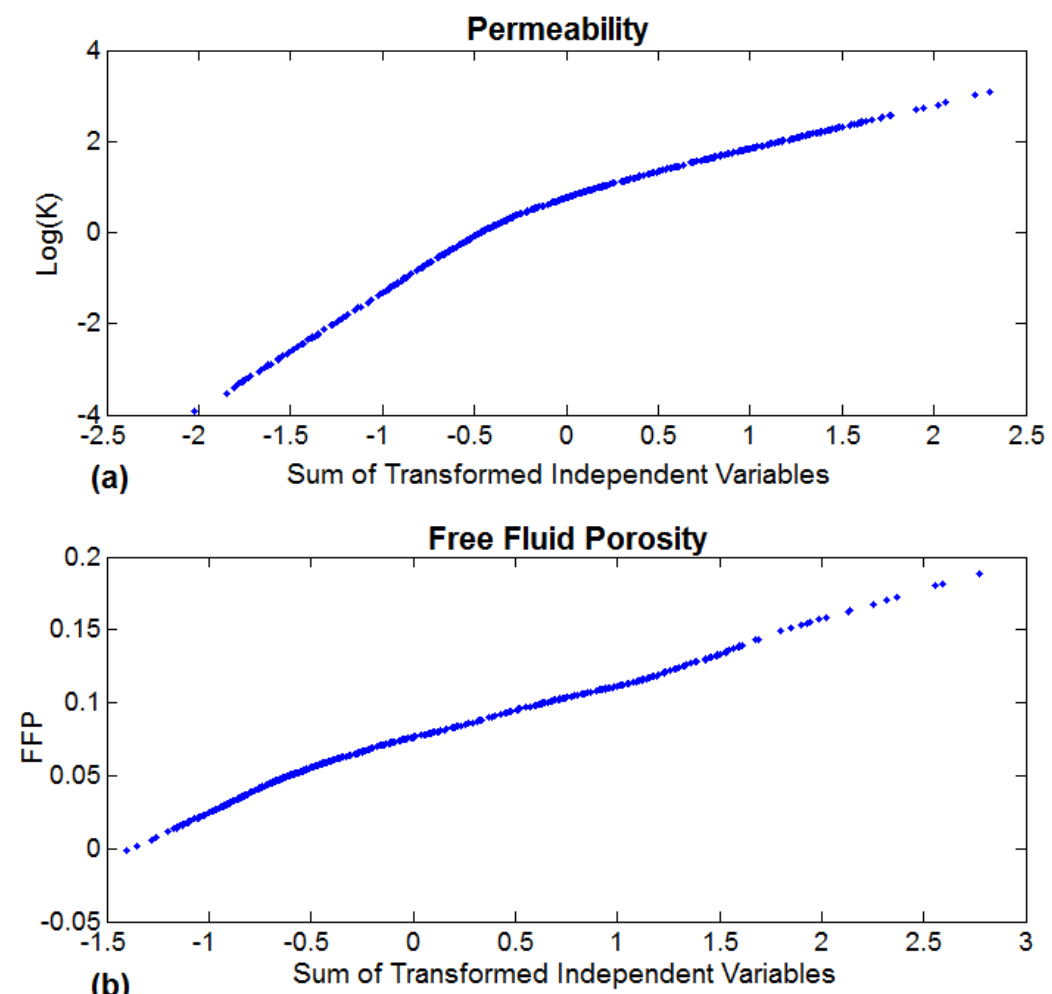

(b)

Fig. 12. Relation between sum of transformed independent variables and: (a) permeability, and (b) free fluid porosity determined by ACE.

Figure 12 shows the relationship between permeability and free fluid porosity and sum of optimal transformation of input parameters. A simple curve fitting can result in permeability and free fluid porosity through following equations.

$$
\log (K)=a_{3}\left(\varphi^{*}(\log (K))\right)^{3}+a_{2}\left(\varphi^{*}(\log (K))\right)^{2}+a_{1}\left(\varphi^{*}(\log (K))\right)+a_{0},
$$

where

$$
a_{3}=0.070938496352028 \text {, }
$$$$
a_{2}=-0.380116311467027,
$$
$a_{1}=1.466278287151171$, and $a_{0}=0.727440481067394$.

$$
F F P=a_{3}\left(\varphi^{*}(F F P)\right)^{3}+a_{2}\left(\varphi^{*}(F F P)\right)^{2}+a_{1}\left(\varphi^{*}(F F P)\right)+a_{0},
$$

where

$$
a_{3}=0.003322744745387 \text {, }
$$$$
a_{2}=-0.007556364428498 \text {, }
$$
$a_{1}=0.040164861128829$, and $a_{0}=0.076864737700259$. 


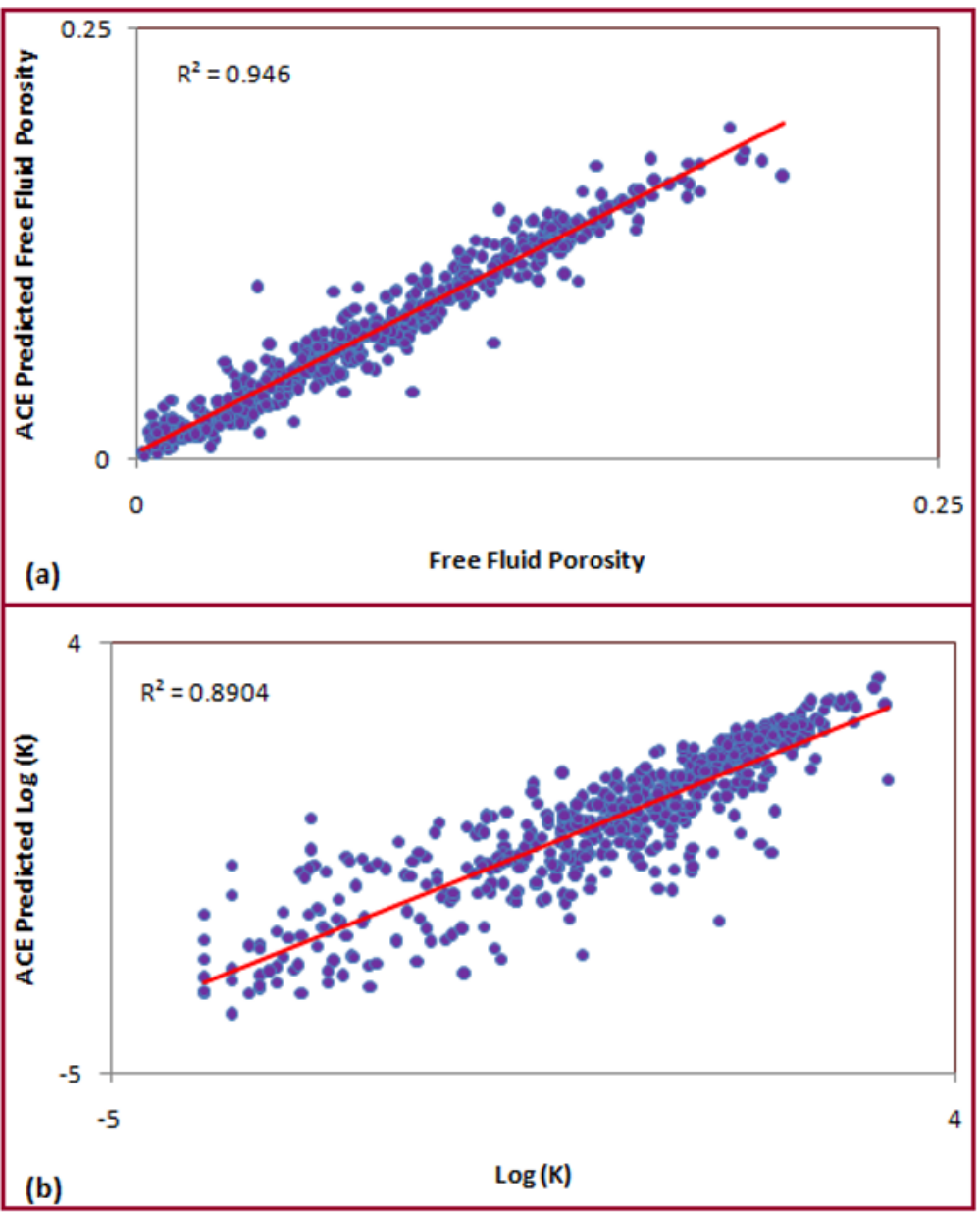

Fig. 13. Crossplots showing correlation coefficient between measured and predicted: (a) free fluid porosity, and (b) $\log (\mathrm{K})$.

After developing above formulation through the use of ACE method, performance of constructed model was assessed by means of test data. Figure 13 shows correlation coefficient between measured and predicted NMR parameters. This figure shows that the ACE model was capable of producing high accuracy results. For better illustration, results of ACE models are compared with individual models viz. neural network, fuzzy logic, optimized neural network, and optimized fuzzy logic in term of correlation coefficient and mean square error (Fig. 14). This figure shows that ACE is a good alternative for combining different models in order to enhance accuracy of final prediction. 

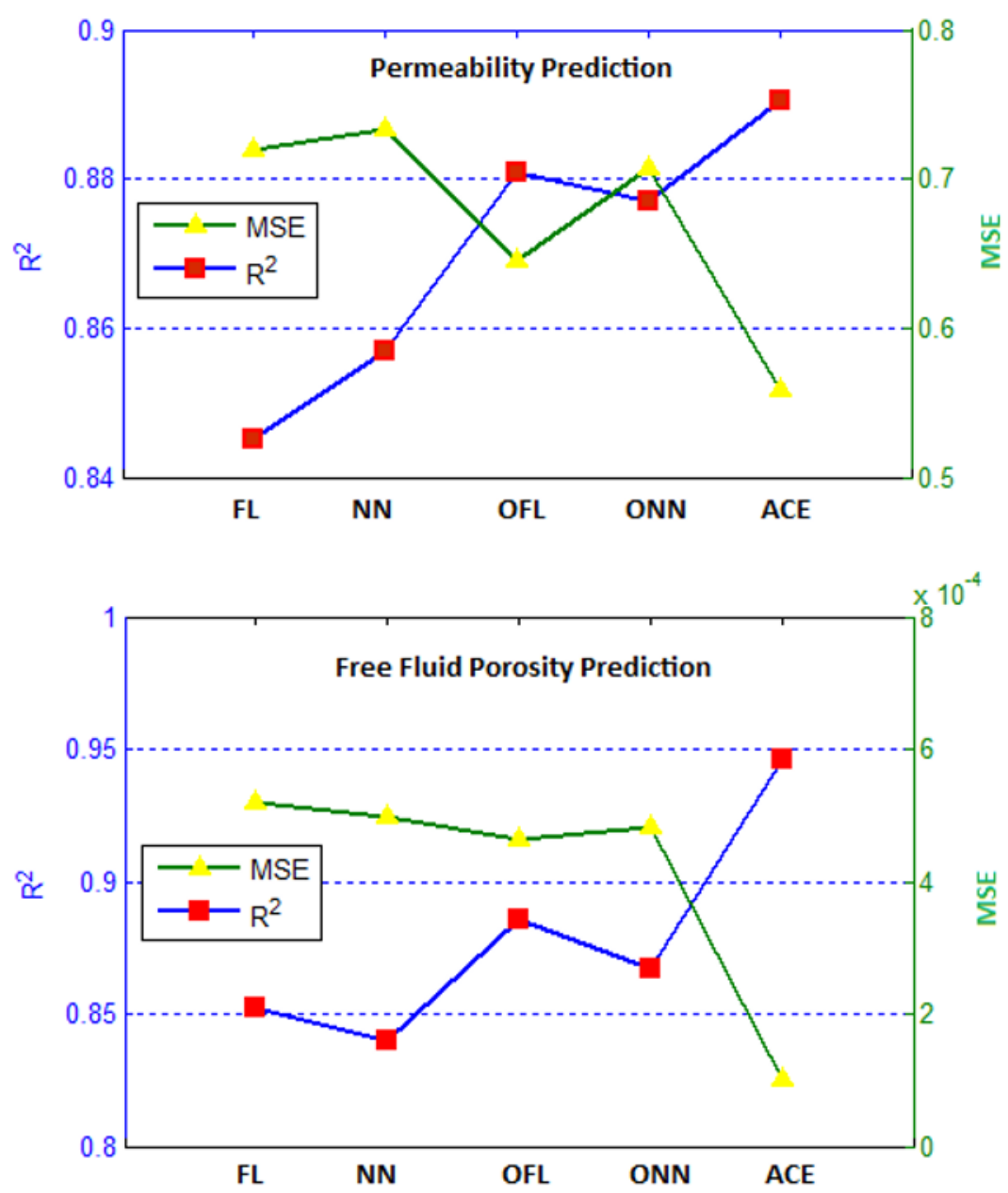

Fig. 14. A comparison between different models versus correlation coefficient and mean square error.

\section{CONCLUSIONS}

In this study, a stochastic optimization tool, called hybrid genetic algorithmpattern search, was employed to strengthen fuzzy logic and artificial neural network. Strengthened models were then used to estimate nuclear magnetic resonance (NMR) log parameters, including free fluid porosity and rock permeability. At the final step of this study, an ACE committee with optimized and non-optimized models was constructed. Some noticeable points deduced from this study can be expressed as follows: 
- In spite of complex behavior of carbonate reservoirs, neural network and fuzzy logic are able to extract the formulation between conventional well log data and NMR log parameters, including free fluid porosity and permeability.

- Performance of back-propagation neural network is greatly dominated by initial weights and biases which are randomly assigned to the neural network. Furthermore, the back-propagation algorithm is highly at risk of trapping in local minima.

- Fuzzy clustering is strongly governed by heterogeneity of training data. This flaw might result in non-smooth multi-dimensional formulation. Therefore, need of comprehensive training database is inevitable.

a Subtractive clustering has just some control on means of Gaussian membership functions, i.e., no modification on variances (spread) of membership functions is done by it.

- Hybrid genetic algorithm-pattern search as a global optimization tool provides a stochastic search capability which is not affected by initial condition of algorithm run. It converges to global minimum regardless from where it starts.

- Extracting optimal weights and biases of neural network through the use of stochastic search of hybrid genetic algorithm-pattern search tool is more confident and efficient than the use of back-propagation algorithm.

a By extracting fuzzy formulation through the use hybrid genetic algorithm-pattern search tool, it is possible to have a control on both means and variances (spread) of Gaussian membership functions.

a Stochastically optimized fuzzy logic and neural network performed better than non-optimized ones.

a Convergence speed of hybrid genetic algorithm-pattern search is low. Substituting faster algorithms for future works are suggested.

- ACE committee machine with optimized and non-optimized models can significantly enhance accuracy of final prediction.

a In situations where there are multiple choices to solve a problem, by little additional computation it is possible to construct an ACE committee machine to improve precision of prediction.

- Implementation of the propounded strategy provides an accurate, quick and cost-effective way of estimating NMR log parameters, including free fluid porosity and rock permeability from conventional well log data for wells that have no NMR run. 


\section{Appendix}

\section{Conversion factors for transferring permeability and sonic transit time units from field units to SI units}

\begin{tabular}{|c|c|c|}
\hline \multirow{2}{*}{ Parameter } & \multicolumn{2}{|c|}{ Conversion factor } \\
\cline { 2 - 3 } & Field units & SI units \\
\hline Permeability & mili-darcy $[\mathrm{md}]$ & $=9.86923 \times 10^{-4} \mu \mathrm{m}^{2}$ \\
Sonic transit time & microsecond per foot $[\mu \mathrm{s} / \mathrm{ft}]$ & $=3.289474 \times 10^{-6} \mathrm{~s} / \mathrm{m}$ \\
\hline
\end{tabular}

\section{References}

Afshar, M., A. Gholami, and M. Asoodeh (2014), Genetic optimization of neural network and fuzzy logic for oil bubble point pressure modeling, Korean $J$. Chem. Eng. 31, 3, 496-502, DOI: 10.1007/s11814-013-0248-8.

Al-Jarrah, O.M., and A. Halawani (2001), Recognition of gestures in Arabic sign language using neuro-fuzzy systems, Artif. Intell. 133, 1-2, 117-138, DOI: 10.1016/S0004-3702(01)00141-2.

Asoodeh, M. (2013), Prediction of Poisson's ratio from conventional well log data: A committee machine with intelligent systems approach, Energ. Source. A 35, 10, 962-975, DOI: 10.1080/15567036.2011.557693.

Asoodeh, M., and P. Bagheripour (2012a), Prediction of compressional, shear, and stoneley wave velocities from conventional well log data using a committee machine with intelligent systems, Rock Mech. Rock Eng. 45, 1, 45-63, DOI: 10.1007/s00603-011-0181-2.

Asoodeh, M., and P. Bagheripour (2012b), Estimation of bubble point pressure from PVT data using a power-law committee with intelligent systems, J. Petrol. Sci. Eng. 90-91, 1-11, DOI: 10.1016/j.petrol.2012.04.021.

Asoodeh, M., and P. Bagheripour (2013a), Core porosity estimation through different training approaches for neural network: Back-propagation learning vs. genetic algorithm, Int. J. Comput. Appl. 63, 5, 11-15, DOI: 10.5120/104615172.

Asoodeh, M., and P. Bagheripour (2013b), Fuzzy classifier based support vector regression framework for Poisson ratio determination, J. Appl. Geophys. 96, 7-10, DOI: 10.1016/j.jappgeo.2013.06.006. 
Asoodeh, M., and P. Bagheripour (2013c), Neuro-fuzzy reaping of shear wave velocity correlations derived by hybrid genetic algorithm-pattern search technique, Cent. Eur. J. Geosci. 5, 2, 272-284, DOI: 10.2478/s13533-012-0129-4.

Asoodeh, M., and K. Kazemi (2013), Estimation of bubble point pressure: Using a genetic integration of empirical formulas, Energ. Source. A 35, 12, 11021109, DOI: 10.1080/15567036.2011.574195.

Asoodeh, M., A. Gholami, and P. Bagheripour (2014a), Oil- $\mathrm{CO}_{2}$ MMP determination in competition of neural network, support vector regression, and committee machine, J. Disper. Sci. Technol. 35, 4, 564-571, DOI: 10.1080/01932691. 2013.803255.

Asoodeh, M., A. Gholami, and P. Bagheripour (2014b), Asphaltene precipitation of titration data modeling through committee machine with stochastically optimized fuzzy logic and optimized neural network, Fluid Phase Equilibr. 364, 67-74, DOI: 10.1016/j.fluid.2013.12.016.

Bagheripour, P., and M. Asoodeh (2013), Fuzzy ruling between core porosity and petrophysical logs: Subtractive clustering vs. genetic algorithm-pattern search, J. Appl. Geophys. 99, 35-41, DOI: 10.1016/j.jappgeo.2013.09.014.

Barhen, J., M. Zak, and S. Gulati (1989), Fast neural learning algorithms using networks with non-Lipschitizian dynamics. In: J.C. Rault (ed.), Proceedings of Neuro-Nimes '89, EC2 Press, Paris, 55-68.

Bataineh, K.M., M. Naji, and M. Saqer (2011), A comparison study between various fuzzy clustering algorithms, Jordan J. Mech. Ind. Eng. 5, 4, 335-343.

Breiman, L., and J.H. Friedman (1985), Estimating optimal transformations for multiple regression and correlation, J. Am. Stat. Assoc. 80, 391, 580-598, DOI: 10.1080/01621459.1985.10478157.

Chen, C.H., and Z.S. Lin (2006), A committee machine with empirical formulas for permeability prediction, Comput. Geosci. 32, 4, 485-496, DOI: 10.1016/ j.cageo.2005.08.003.

Conn, A.R., N.I.M. Gould, and P. Toint (1991), A globally convergent augmented Lagrangian algorithm for optimization with general constraints and simple bounds, SIAM J. Numer. Anal. 28, 2, 545-572, DOI: 10.1137/0728030.

Gholami, A., S. Moradi, and B. Dabir (2013), A power law committee scaling equation for quantitative estimation of asphaltene precipitation, Int. J. Sci. Emerging Technol. 6, 5, 275-283.

Gupta, M.M., and H. Ding (1994), Foundations of fuzzy neural computations. In: F. Aminzadeh and M. Jamshidi (eds.), Soft Computing: Fuzzy Logic, Neural Networks, and Distributed Artificial Intelligence, Prentice Hall Inc., Upper Saddle River, 165-199.

Hagan, M.T., and M.B. Menhaj (1994), Training feedforward networks with the Marquardt algorithm, IEEE Trans. Neural Netw. 5, 6, 989-993, DOI: 10.1109/72.329697. 
Kadkhodaie-Ilkhchi, A., M.R. Rezaee, and S.A. Moallemi (2006), A fuzzy logic approach for the estimation of permeability and rock type from conventional well log data: An example from the Kangan reservoir in the Iran offshore gas field, J. Geophys. Eng. 3, 4, 356-369, DOI: 10.1088/1742-2132/3/4/007.

Kadkhodaie-Ilkhchi, A., H. Rahimpour-Bonab, and M.R. Rezaee (2009), A committee machine with intelligent systems for estimation of total organic carbon content from petrophysical data: An example from Kangan and Dalan reservoirs in South Pars gas field, Iran, Comput. Geosci. 35, 3, 459-474, DOI: 10.1016/j.cageo.2007.12.007.

Labani, M.M., A. Kadkhodaie-Ilkhchi, and K. Salahshoor (2010), Estimation of NMR log parameters from conventional well log data using a committee machine with intelligent systems: A case study from the Iranian part of the South Pars gas field, Persian Gulf Basin, J. Petrol. Sci. Eng. 72, 1-2, $175-$ 185, DOI: 10.1016/j.petrol.2010.03.015.

Lippmann, R.P. (1987), An introduction to computing with neural nets, ASSP Mag. IEEE 4, 2, 4-22, DOI: 10.1109/MASSP.1987.1165576.

Malallah, A., R. Gharbi, and M. Algharaib (2006), Accurate estimation of the world crude oil PVT properties using graphical alternating conditional expectation, Energy Fuels 20, 2, 688-698, DOI: 10.1021/ef0501750.

Malki, H.A., and J. Baldwin (2002), A neuro-fuzzy based oil/gas producibility estimation method. In: Proc. Int. Jt. Conf. Neural Networks IJCNN 2002, Vol. 1, 12-17 May 2002, Honolulu, USA, 896-901, DOI: 10.1109/IJCNN. 2002.1005593.

Mathworks (2011), MATLAB user's guide. Fuzzy logic, neural network \& GA and direct search toolboxes, Mathworks, Inc., CD-rom.

Mohaghegh, S. (2000a), Virtual-intelligence applications in petroleum engineering: Part 1 - Artificial neural networks, J. Petrol. Technol. 52, 9, 64-73, DOI: 10.2118/58046-JPT.

Mohaghegh, S. (2000b), Virtual-intelligence applications in petroleum engineering: Part 2 - Evolutionary computing, J. Petrol. Technol. 52, 10, 40-46, DOI: 10.2118/61925-JPT.

Mohaghegh, S. (2000c), Virtual-intelligence applications in petroleum engineering: Part 3 - Fuzzy logic, J. Petrol. Technol. 52, 11, 82-87, DOI: 10.2118/ 62415-JPT.

Ogilvie, S.R., S. Cuddy, C. Lindsay, and A. Hurst (2002), Novel methods of permeability prediction from NMR tool data, Dialog, London Petrophysical Society, London, 1-14.

Sharkey, A.J.C. (1996), On combining artificial neural nets, Connect. Sci. 8, 3-4, 299-314, DOI: 10.1080/095400996116785.

Shokir, E.M. (2007), $\mathrm{CO}_{2}$-oil minimum miscibility pressure model for impure and pure $\mathrm{CO}_{2}$ streams, J. Petrol. Sci. Eng. 58, 1-2, 173-185, DOI: 10.1016/ j.petrol.2006.12.001. 
Xue, G., A. Datta-Gupta, P. Valko, and T. Blasingame (1996), Optimal transformations for multiple regression: application to permeability estimation from well logs, Paper No. SPE-35412, Society of Petroleum Engineers, Inc., Richardson, USA.

Zadeh, L.A. (1965), Fuzzy sets, Inf. Control. 8, 3, 338-353, DOI: 10.1016/S00199958(65)90241-X.

Received 2 February 2014 Received in revised form 5 April 2014 Accepted 9 May 2014 